\title{
SENSORIMOTOR ADAPTATION OF WHOLE-BODY POSTURAL CONTROL
}

\author{
DOUGLAS M. SHILLER, ${ }^{\mathrm{a}, \mathrm{b}, \mathrm{c} *}$ \\ LOUIS-NICOLAS VEILLEUX, ${ }^{\mathrm{b}, \mathrm{c}, \mathrm{d}, \mathrm{e}}$ MIKAËL MAROIS, ${ }^{\mathrm{b}, \mathrm{c}, \mathrm{f} \dagger}$ \\ LAURENT BALLAZ ${ }^{\mathrm{b}, \mathrm{c}, \mathrm{g}}$ AND MARTIN LEMAY ${ }^{\mathrm{b}, \mathrm{c}, \mathrm{g}}$ \\ ${ }^{a}$ École d'orthophonie et d'audiologie, Université de Montréal, \\ C.P. 6128, succursale Centre-ville, Montréal, Québec H3C 3J7, \\ Canada \\ ${ }^{\mathrm{b}}$ Centre Hospitalier Universitaire Sainte-Justine Research Centre, \\ 3175 Chemin de la Côte-Sainte-Catherine, Montreal, Quebec H3T \\ 1C4, Canada \\ ${ }^{\mathrm{C}}$ Centre de Réadaptation Marie Enfant, 5200 Rue \\ Bélanger, Montréal, Quebec H1T 1C9, Canada \\ d Département de kinésiologie, Université de Montréal, 2100 \\ boulevard Édouard-Montpetit, Bureau 8202, Montreal, Quebec \\ H3T 1J4, Canada \\ ${ }^{\text {e }}$ Hôpital Shriners pour Enfant-Canada, 1003 Boulevard \\ Décarie, Montréal, Quebec H4A OA9, Canada \\ ${ }^{\mathrm{f}}$ Département de Génie Physique, Polytechnique Montréal, \\ 2900 boul. Édouard-Montpetit, Montréal, Quebec H3T 1J4, Canada \\ ${ }^{9}$ Département des sciences de l'activité physique, Université \\ du Québec à Montréal, C.P. 8888, succursale Centre-Ville, \\ Montreal, Quebec H3C 3P8, Canada
}

\begin{abstract}
The aim of the present study was to examine the modification of postural symmetry during quiet standing using a sensorimotor adaptation paradigm. A group of neurologically typical adult participants performed a visually guided mediolateral (left-right) weight shifting task requiring precise adjustments in body orientation. During one phase of the task, the visual feedback of center of pressure (COP) was systematically biased toward the left or the right, requiring an adjustment in posture to compensate. COP during quiet standing without visual feedback was examined prior to and immediately following the sensorimotor adaptation procedure, in order to observe whether compensatory adjustments in postural control resulting from the visualfeedback manipulation would transfer to the control of whole-body COP during quiet standing. Results showed that the sensorimotor adaptation procedure induced a small but reliable compensatory change in the stance of participants, resulting in a change in postural symmetry and control that was found to persist even after normal visual feedback was restored. (C) 2017 IBRO. Published by Elsevier Ltd. All rights reserved.
\end{abstract}

\footnotetext{
"Correspondence to: D. M. Shiller, École d'orthophonie et d'audiologie, Université de Montréal, C.P. 6128, succursale Centre-ville, Montréal, Québec H3C 3J7, Canada.

E-mail address: douglas.shiller@umontreal.ca (D. M. Shiller).

Present address for Mikaël Marois: Thayer School of Engineering, Dartmouth College, 14 Engineering Drive, Hanover, NH 03755, United States.

Abbreviations: ANOVAs, analysis of variance; COP, center of pressure.
}

Key words: motor learning, postural control, sensorimotor adaptation, visual feedback, center of pressure.

\section{INTRODUCTION}

In the paradigm of sensorimotor adaptation, sensory feedback (typically proprioceptive or visual) is altered in near-real-time during a period of motor practice, and compensatory changes in movement parameters are evaluated. This adaptive change in motor function is characterized by a gradual improvement in performance over repeated practice trials and persisting beyond the period of the feedback perturbation, indicating that motor learning has occurred (see Shadmehr et al., 2010, for review). In current models of sensorimotor control, this form of sensory-based motor learning is believed to be driven, on a trial-by-trial basis, by an improvement in the accuracy of a predictive internal forward model used to estimate the sensory consequences of actions - a process presumed to be central to sensory-motor planning and control (Shadmehr et al., 2010).

A number of studies of sensorimotor adaptation have been carried out examining upper-limb pointing movements in healthy participants. These studies have involved visual manipulations of hand position (e.g., prismatic adaptation) or externally applied force-fields, both of which alter the relation between motor planning and the resulting, perceived movement (e.g., Nakajima, 1988; Shadmehr and Mussa-Ivaldi, 1994; Bhushan et al., 2000; Martin et al., 2002; Kennedy and Raz, 2005; Pisella et al., 2006; Veilleux and Proteau, 2015). Motor adaptation to perturbations in gait patterns has also been examined using split-belt treadmills to differentially perturb walking speed in the two legs (e.g., Reisman et al., 2005) or circular treadmills which require a curved walking pattern (e.g., Gordon et al., 1995; Weber et al., 1998). Sensorimotor adaptation studies have also been carried out using real-time alterations in auditory feedback during the control of speech production (Houde and Jordan, 1998), demonstrating that following speech practice under feedback-altered conditions, talkers adjust their oral motor output in order to reduce the perceived magnitude of the perturbation (e.g., Houde and Jordan, 1998; Shiller et al., 2009). The results of these studies show that the neural control of motor behavior is capable of adapting to varying sensorimotor conditions across a wide range of tasks and modalities.

The aim of the present study is to examine whether a real-time manipulation of sensory feedback related to postural motor control will similarly result in a 
recalibration of sensorimotor control processes. A pair of studies has previously demonstrated changes in postural symmetry in association with the adaptation of upper limb reaching movements to a visual horizontal prism (using glasses to suddenly shift the entire visual field to the right or left relative to the participant; Tilikete et al., 2001; Michel et al., 2003). The authors of these studies have suggested that adaptation to such global visuospatial perturbations may have been due to changes in participants' higher level cognitive representation of external space relative to the body, rather than a recalibration of sensorimotor processes per se (Tilikete et al., 2001; Michel et al., 2003). The present study avoids such complications in interpretation arising from prismatic visual shifts by altering visual feedback of a specific postural variable without altering the visual representation of the world relative to the participant, combined with a postural movement task that focused specifically on changes in weight distribution.

The control of whole-body posture involves the integration and processing of somatosensory, vestibular and visual feedback in order to stabilize the body and minimize sway (Fitzpatrick and McCloskey, 1994). The center of mass of the body is maintained over the supporting base through changes in the center of pressure (COP), which corresponds to the point of application of the ground reaction force vector. The COP of a participant standing with both feet touching the ground is generally found at a central location between the feet (Winter, 1995). In patients with unilateral musculoskeletal or neurological deficits, however, postural asymmetry tends to result in COP deviating from the central region of the supporting base (Shumway-Cook and Woollacott, 2007). More weight is maintained on the non-involved leg in such patients, which affects the control of gait and posture (Ring and Mizrahi, 1991). For example, weight-bearing asymmetry in stroke patients increases mediolateral sway (Marigold and Eng, 2006) and synchronization of COP between legs during standing (Mansfield et al., 2011), and affects the symmetry of time spent on each leg during gait (Hendrickson et al., 2014). Without correcting their postural asymmetry, such patients also maintain an elevated long-term risk of falling and back-pain (Di Fabio and Badke, 1990), and a patient's capacity for sensorybased motor adaptation provides a potentially important mechanism for such a behavioral correction.

Before considering such an approach for patients with postural asymmetry, it is necessary to validate whether a visually guided postural control task can be used to induce sensorimotor adaptation in whole-body posture. In the present study, a group of neurotypical participants performed a visually guided medio-lateral weight shifting task requiring precise adjustments in body orientation. During a portion of the task, the visual feedback of participants' COP was systematically biased toward the right or left (with half of the participants in each condition), requiring an adjustment in postural control to compensate. Center of pressure during quiet standing (without visual feedback) was examined prior to and following the sensorimotor adaptation procedure, in order to observe whether compensatory adjustments in postural control resulting from the visual-feedback manipulation would transfer to the control of whole-body COP during quiet standing, lasting beyond the period of altered feedback.

\section{EXPERIMENTAL PROCEDURES}

\section{Participants and experimental methods}

Twenty-two participants (age 20-33 years), with no reported history of neurological, vestibular, sensory or motor disorder were tested. Participants were instructed to stand quietly on a force platform (Accugait, Advanced Mechanical Technology, USA) with the feet at shoulder width and the arms held at the sides. Visual markers were placed around both feet in order to ensure that the same foot position was maintained for the duration of the task.

The primary task involved a visually guided postural movement involving a lateral displacement of COP to the left or right. Visual feedback of COP location was presented on a computer display $\left(46^{\prime \prime}\right.$, positioned at a distance of $2 \mathrm{~m}$ ), which included a central rectangular red region that represented the "home" position, two red "target" rectangular areas (corresponding to an $8-\mathrm{cm}$ change in COP on the force plate, and located $16 \mathrm{~cm}$ to the right and left of the home position on the screen), and a small black filled circle (1-cm diameter) that represented the current COP location (Fig. 1). Participants were familiarized with the visual interface during a short practice period $(\sim 1 \mathrm{~min})$ in which they were allowed to freely alter their stance, thereby moving the on-screen representation of COP. During the subsequent postural movement phases of the experiment (Baseline, Adaptation and Washout) the participants' task on each trial was as follows: (1) maintain their COP in the central region for at least $3 \mathrm{~s}$, (2) carry out a COP movement to the right or left target, (3) maintain the COP location within the target area for $2 \mathrm{~s}$, and (4) move the COP back to the central region. The left or right target to which participants had to move on each trial was indicated by the presence of a green border region around the red rectangular target area (Fig. 1). Similarly, a green rectangle around the central "home" region indicated when the participant was to return from the target area back to the center base. Participants were instructed to move their COP immediately upon seeing the visual cue. On average, the duration of the COP displacement was 1.38 (0.34 SD) seconds for the right-bias group and 1.54 (0.42 SD) seconds for the left-bias group.

The experimental protocol involved four phases: (1) the Baseline phase (30 movements) during which postural movements were carried out with "normal" (i.e., unbiased) visual feedback of COP position; (2) the Adaptation phase (120 movements) in which postural movements were carried out under conditions of altered visual feedback, (3) the No-feedback phase (10 movements) in which postural movements were carried out without any visual feedback, and (4) the Wash-out phase (30 movements), during which postural movements were carried out once again under 


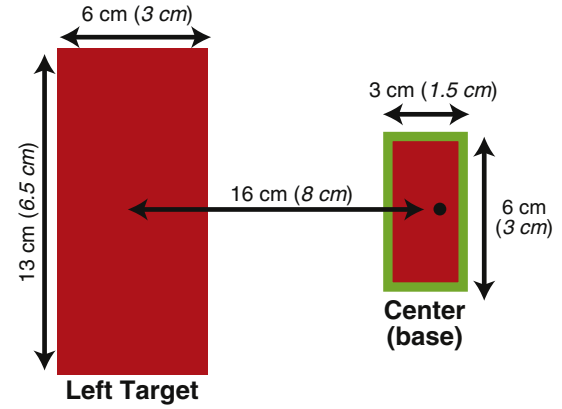

Fig. 1. Positions of the center base and the target regions on screen. The distances are presented in units of the on-screen visual representation and, in italics, the corresponding COP displacement on the force plate. The black dot near the center shows the current COP location.

conditions of normal (unaltered) visual feedback. Participants carried out the 190 movements during the four different phases consecutively. Adjustments in postural symmetry resulting from the alteration of visual feedback (in the Adaptation and Wash-out phases) were further examined at three key time-points using a quiet standing task in which participants stood still on the force plate for one minute while visually fixating an onscreen target. The three time-points were: (1) immediately preceding the Adaptation phase (StandingBaseline); (2) immediately after the Adaptation phase (Standing-Post-Adaptation); and (3) immediately after the Washout phase (Standing-Post-Washout). The participants were randomly assigned to one of two groups ( $n=11$ in each group): one involving a left visual bias during the Adaptation phase (Left-bias group), and one involving a right visual bias (Right-bias group).

During the 120-trial Adaptation phase, a bias to the right or left (depending on group membership) was introduced in the relation between the visual display and the participant's COP. This visual bias was linearly increased over the course of the first 60 movements, reaching a peak of $3 \mathrm{~cm}$, at which point, the actual location of the participant's COP was $3 \mathrm{~cm}$ to the right (right-bias group) or left (left-bias group) of the COP position represented on the visual display. Note that this had the effect of making the participant appear to be leaning too far to the left (for the right-bias group) or too far to the right (for the left-bias group), requiring a postural adjustment toward the right or left (respectively) to compensate. This $3-\mathrm{cm}$ bias was subsequently maintained for 60 additional COP movements during the Adaptation phase.

The No-feedback phase consisted of COP displacements during which the on-screen representation of the participant's COP location was not visible. Under these conditions, participants carried out 10 COP movements (five to the left and five to the right, in a randomized sequence) that were meant to match the displacements carried out during the Adaptation phase. The COP displacements without visual feedback allowed us to test whether any compensatory adjustments observed during the Adaptation phase in fact depended directly upon the continual availability of visual feedback (i.e., closed-loop control), or whether such adjustments reflect a change in feed-forward planning. Finally, during the Wash-out phase, participants carried out 30 trials under conditions of normal visual feedback (same as the Baseline condition), during which the adjustments in postural control observed during the Adaptation phase would be unlearned.

\section{Data analyses}

Ground reaction force was sampled at $50 \mathrm{~Hz}$ and lowpass filtered at $6 \mathrm{~Hz}$ (second-order, zero phase Butterworth filter Matlab v. 7.0, Mathworks, Natick, MA) prior to calculating COP in the mediolateral (left-right) and anteroposterior (front-back) dimensions. Note that on the mediolateral axis, more positive values are toward the right while on the anteroposterior axis, more positive values are toward the front.

An examination of postural control during the Baseline, Adaptation, No-feedback and Wash-out phases (i.e., the procedures involving target-directed changes in COP) focused on the participants' mediolateral COP position at the center base location, which served as the starting position for the center-out movements (i.e., away from the center base), and as the target position for the out-center movements (toward the center base). This focus on the center base location allowed us to characterize the changes in postural control that accompanied these "dynamic" phases of the sensorimotor adaptation procedure, while maintaining our focus on the participant's representation of the center (midline) position critical to the quiet standing task.

Unlike during point-to-point arm movements, in which the limb can begin and end with the arm nearly at rest relative to the torso, whole-body standing requires a continuous process of sensory-based (or predictive model-based) motor adjustments to maintain balance (Shumway-Cook \& Woollacott, 2012; Winter, 1995; Morasso and Schieppati, 1999), resulting in a degree of sway at all times (even during the maintenance of the target or starting position). In order to distinguish this habitual postural sway from the target-directed changes in COP, each COP movement start and end was identified operationally as the first zero-crossing in COP velocity immediately preceding (for movement onset) or following (for movement offset) the large velocity peak associated with the target-directed COP movement (see Fig. 2). The separate analysis of the movement onsets (for center-out) and movement offsets (for out-center) allows for the characterization of adaptive changes in the participants' representation of body posture at two functionally distinct moments during the COP displacement task. As such, similar patterns of positional bias observed for both movement onsets and offsets would strengthen the idea that such changes were robustly represented in participants' postural control.

To assess changes in the control of the leftward and rightward COP movements under the various feedback phases (Baseline, Adaptation, No-feedback and Washout), we examined COP position associated with 

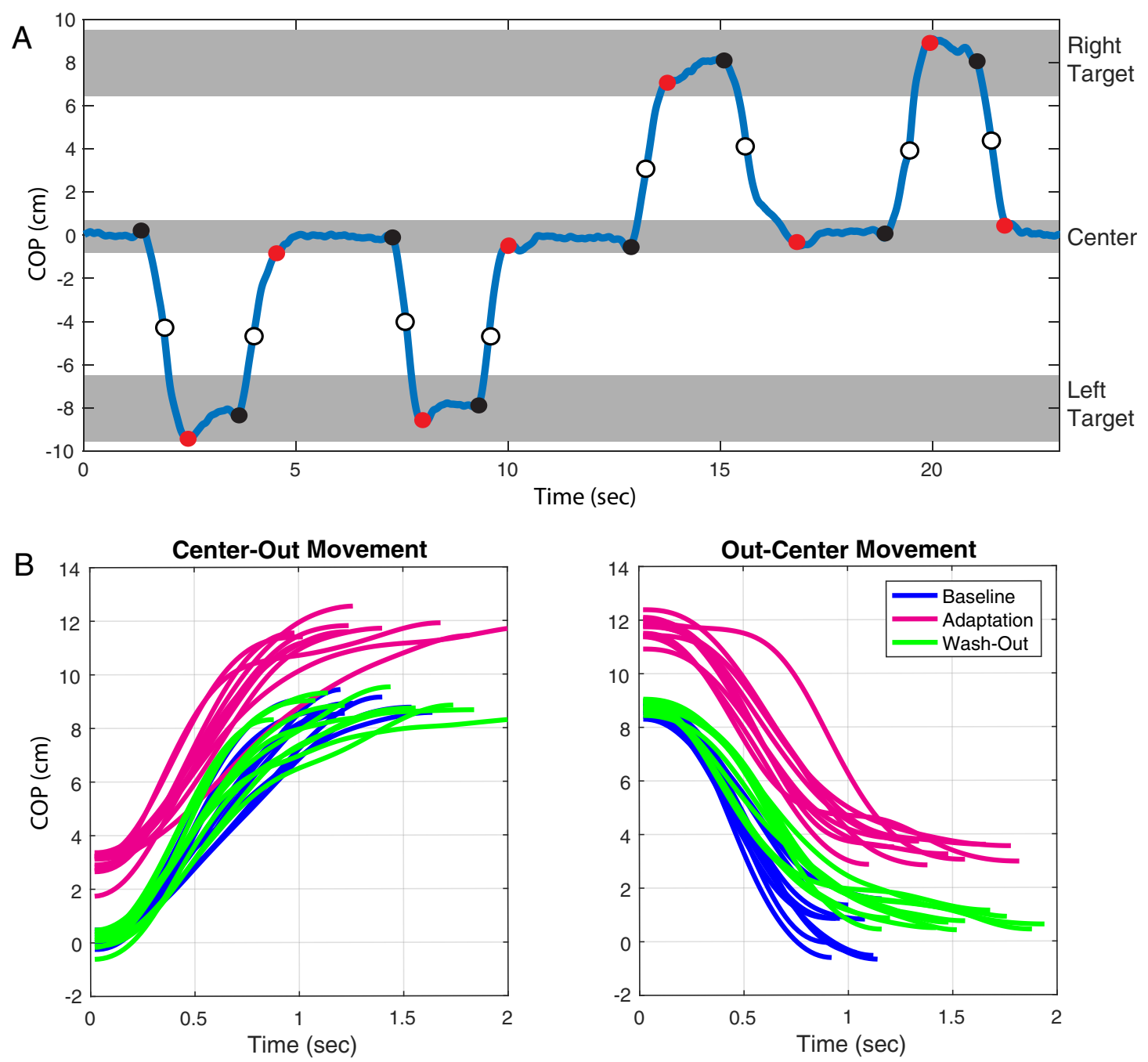

Fig. 2. Example COP trajectories for one participant. A) Mediolateral COP trace during the Baseline phase. Four successive movements are shown from the center position (gray area centered at $0 \mathrm{~cm}$ ) toward the target location to the left (negative direction) or to the right (positive direction) and then back to the center. Open circles correspond to the time of peak velocity for each movement. Black solid dots correspond to the first zerocrossing in velocity prior to peak velocity (defining movement onset). Red dots correspond to the first zero-crossing in velocity following peak velocity (defining movement offset). B) Example COP traces from one participant in the right-bias group, showing center-out movements toward the right target (left panel) and out-center movements from the right target (right panel) at the end of the Baseline phase (blue), Adaptation phase (magenta) and Wash-out phase (green). (For interpretation of the references to colour in this figure legend, the reader is referred to the web version of this article.)

movement onset and offset at the end of each phase (averaging over the final 10 movements in each phase). For simplicity, the analysis focused on movement onset for center-out movements, and movement offset for outcenter movements (hence, at the center target location). In order to most clearly represent the changes in COP associated with the changing feedback conditions, the COP values at each phase were first normalized by subtracting each participant's mean baseline COP position at the center-base location (to eliminate the contribution of any differences in baseline COP between participants). Mean normalized COP, reflecting the change from baseline, was then examined at each of the three remaining phases (Adaptation, No-feedback and Washout phases) and each movement type (centerout, where the center-base corresponds to the movement start position, and out-center, where the center-base corresponds to the movement end position) using a mixed-factorial ANOVA, with GROUP (Left-bias vs. Right-bias) as a between-group factor and movement DIRECTION (to/from the right vs. to/from the left) as a within-group factor. An additional withinsubject factor was also included (TIME) in order to assess the change in COP at different time-points within the Adaptation and Washout phases. Specifically, differences in COP were examined between the late part the phase (final 10 movements) and early part of the phase (the first 10 movements under full visual perturbation for the Adaptation phase, i.e., trial 61-70, and the first 10 movements of normal visual feedback for the Washout phase).

For the three quiet standing trials (Standing-Baseline, Standing-Post-Adaptation and Standing-Post-Washout), COP was averaged over the final $50 \mathrm{~s}$ of the $60 \mathrm{~s}$ 
standing period. The initial 10-s were eliminated in order to avoid possible movement artifact or initial postural adjustments (see, e.g., Alcantara et al., 2012; Pham et al., 2014). The difference between the two groups of participants (right bias vs. left bias) and the three experimental phases were evaluated using a 2-way mixed-factorial ANOVA, carried out separately for the mediolateral and anteroposterior axes. While we predicted effects only in the mediolateral axis (the axis along which the visually guided control tasks were carried out), the anteroposterior axis was included to verify the specificity of the effects. When necessary, repeatedmeasures t-tests with Holm-Bonferroni corrections for multiple comparisons were used as post hoc pair-wise comparisons for all analysis of variance (ANOVAs).

\section{RESULTS}

\section{Change in COP during the Adaptation, no-feedback} and Washout phases

At the end of the Adaptation phase, during which participants performed postural movements under conditions of altered visual feedback, a difference in normalized COP at the center-base position can be clearly observed between the Left- and Right-bias groups (Fig. 3). This difference between bias-directions can be seen at the start position for center-out movements (Fig. 3, left panel), and at the end position for out-center movements (Fig. 3, right panel). The movement end positions (Fig. 2, right panel) also can be seen to exhibit an effect of movement direction, whereby movements originating from the right (i.e., leftward movements) show more positive end-positions (toward the right), and movements originating from the left show more negative end-positions (toward the left). This reflects a trend on the part of participants to bring their visually guided COP movement to an initial stop (i.e., first velocity zero-crossing) in the region of the rectangular center base target closer to the movement start point. It should be noted that the magnitude of this slight undershoot effect (averaging $3.65 \mathrm{~mm}$ from the target midpoint) is considerably smaller than the width of the center-base target $(15 \mathrm{~mm}$, or $\pm 7.5 \mathrm{~mm}$ from the midpoint). Hence, despite this variation, participants ended their main COP movements within the target region.

These effects were confirmed using a set of 3-way ANOVAs (one for center-out movements and one for out-center movements). Highly reliable main effects of visual-bias GROUP (left-bias vs. right-bias) were observed in both cases (center-out: $F[1,20]=196.18$, $p<0.0001$; out-center: $F[1,20]=193.48, p<0.0001$ ). In the case of movement start positions, there was no reliable main effect of movement DIRECTION (to/from the right vs. to/from the left; $F[1,20]=1.01, p=0.33$ ) and no main effect of TIME (early vs. late in the phase: $F[1,20]=0.31, p=0.58)$. Further, there were no significant 2-way interactions $($ GROUP $\times$ DIRECTION: $F$ $[1,20]=0.40, \quad p=0.53 ; \quad$ GROUP $\times$ TIME: $\quad F[1,20]$ $=0.01, p=0.98 ;$ DIRECTION $\times$ TIME: $F[1,20]=0.34$, $p=0.57)$ and no significant 3-way interaction $(F[1,20]$
START Position (center-out movement)

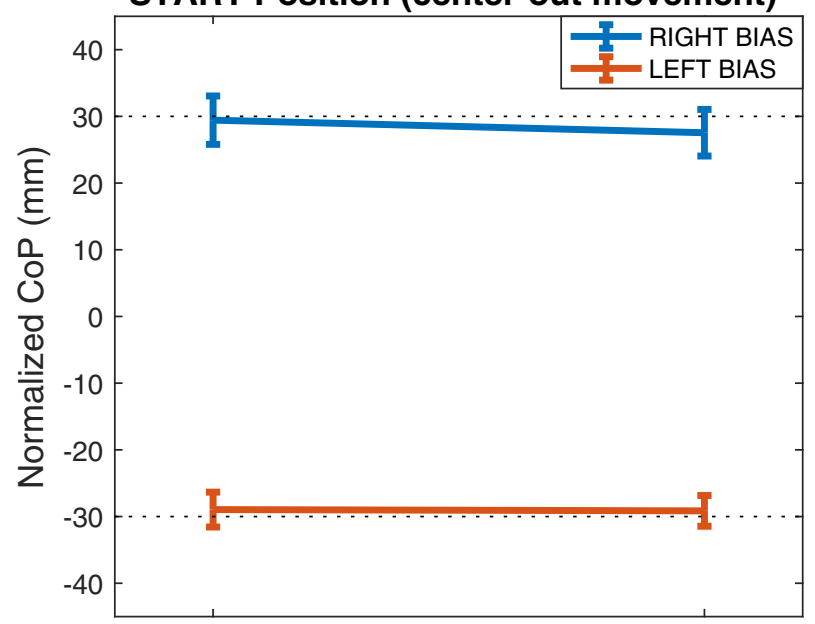

END Position (out-center movement)

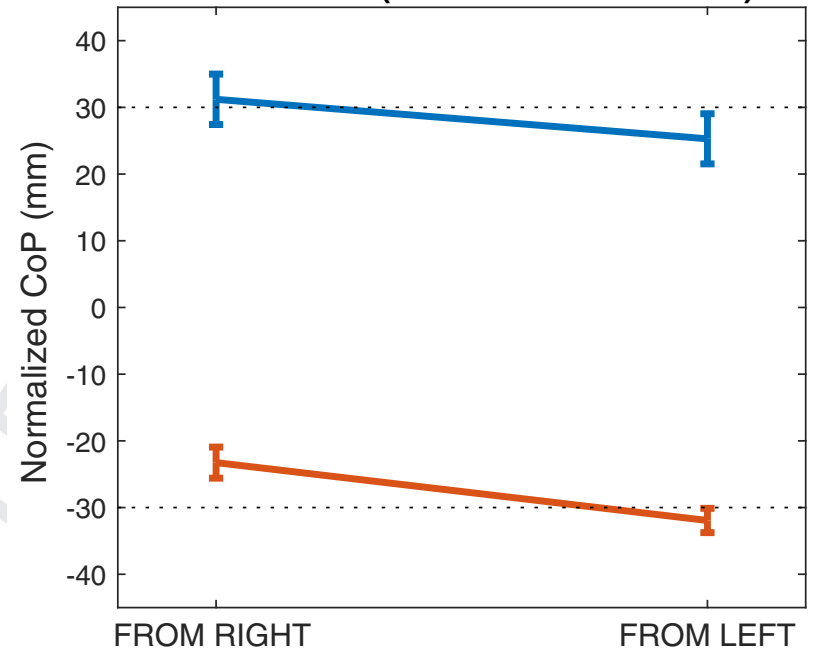

Fig. 3. Mean COP at the end of the Adaptation phase (biased visual feedback): Mean normalized COP position (difference relative to baseline) at the center-base location, observed at the start of centerout movements to the left or right (top panel), and at the end of movements from the right or left (bottom panel). Gray dashed line shows the COP target distance $(30 \mathrm{~mm})$. A clear difference between the Right-bias group (blue line) and Left-bias (red-line) group can be seen for both movement types. An effect of movement direction can also be observed for the movement END positions (bottom panel). Error bars show \pm 1 standard error of the mean. (For interpretation of the references to colour in this figure legend, the reader is referred to the web version of this article.)

$=0.59, p=0.45)$. For the end positions, there was a reliable main effect of movement DIRECTION $(F[1,20]$ $=32.74, p<0.001)$, but again no main effect of TIME $(F[1,20]=0.007, p=0.93)$. Finally, all 2- and 3-way interactions were not significant (GROUP $\times$ DIRECTION: $\quad F[1,20]=2.69, \quad p=0.12 ; \quad$ GROUP $\times$ TIME: $F[1,20]=0.06, p=0.81$; DIRECTION $\times$ TIME: $F$ $[1,20]=3.58, \quad p=0.08 ; \quad 3$-Way: $\quad F[1,20]=0.42$, $p=0.53)$.

While the preceding analysis indicated a difference in COP between the two visual bias conditions (i.e., the main effect of GROUP) at the end of the Adaptation 
phase, it was also of interest whether the change in COP within each of the visual bias conditions was reliably different from 0 (baseline). As the ANOVA showed no interaction between visual-bias group and movement direction, COP start and end positions were collapsed across the two movement directions for this analysis. Using Holm-Bonferroni corrected t-tests, a statistically reliable difference from baseline was found for COP start positions in the right-bias group $(t[10]=8.28$, $p<0.001)$ and left-bias group $(t[10]=-12.09$, $p<0.001$ ). Similarly, a reliable change from baseline was found for COP end positions in the right-bias group $(t[10]=7.98, \quad p<0.001)$ and left-bias group $(t[10]$ $=-13.75, p<0.001)$.

A difference between groups under altered visual feedback conditions indicates that participants successfully used the biased visual feedback to guide their postural movements (i.e., following the instructions for the task). However, the period of No-feedback immediately following the Adaptation phase provided an opportunity to examine whether participants' postural control remained altered even without the use of the biased visual feedback (i.e., sensorimotor learning). Mean normalized COP in the center-base location during this period is shown in Fig. 4 for center-out movements (left panel) and out-center movements (right panel). While the differences from baseline are smaller than those observed during the Adaptation phase, a clear effect of bias-group can still be seen, with the Right-bias group (blue line) positioned to the right (more positive COP) than the Left-bias group (red line). An effect of movement direction can also be observed for the end positions (i.e., the out-center movements; right panel). Here, the COP displacements exhibit a slight overshoot pattern, whereby movements originating from the right showing more negative (leftward) endpositions, and movements originating from the left show more positive (rightward) end-positions. Note, however, that within each of the two bias conditions (blue and red lines), this direction-dependent difference in COP end position (averaging $14.57 \mathrm{~mm}$ ) was comparable to the width of the center-base target region $(15 \mathrm{~mm})$.

The group effect was confirmed using a series of 2way ANOVAs, with reliable main effects of GROUP observed in both cases (center-out movement: $F[1,20]$ $=4.81, \quad p<0.05 ;$ out-center movement: $F[1,20]$ $=4.89, p<0.05)$. As in the Adaptation phase, the center-out movements showed no main effect of movement direction $(F[1,20]=1.45, p=0.24)$ and no GROUP $\times$ DIRECTION interaction $(F[1,20]=0.12$, $p=0.73$ ), while the out-center movements showed a reliable main effect of movement direction $(F[1,20]$ $=7.17 p<0.05)$, with no interaction effect $(F[1,20]$ $=0.43, p=0.51$ ).

While the preceding analysis indicated a reliable difference in COP between the two visual bias groups, it was again of interest to consider the change in COP relative to baseline separately for each of the visual bias conditions. For this more stringent analysis, HolmBonferroni corrected t-tests showed only a marginally reliable difference from baseline for the left-bias group
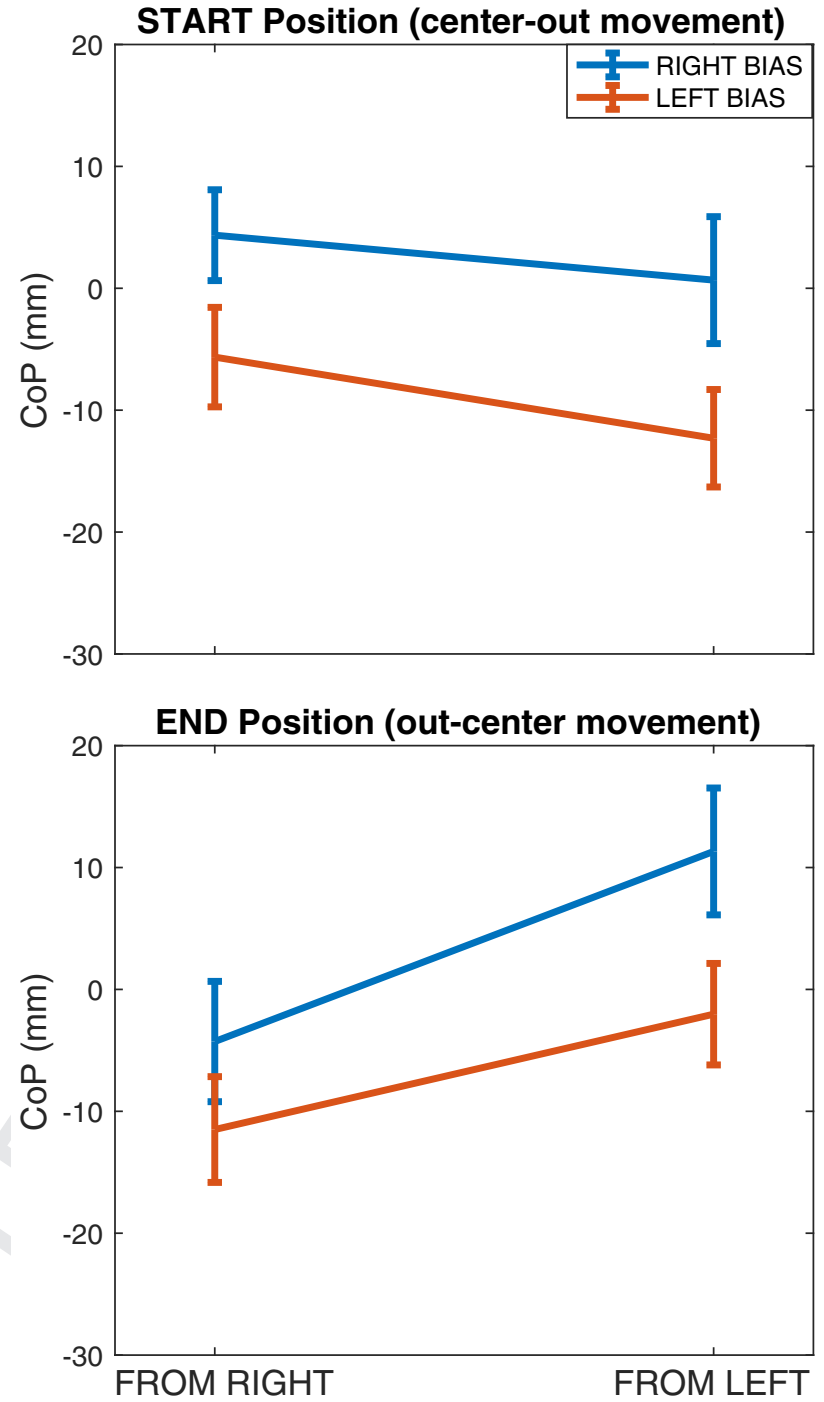

Fig. 4. NO-FEEDBACK PHASE: Mean normalized COP position (difference relative to baseline) at the center-base location, observed at the start of center-out movements to the left or right (top panel), and at the end of movements from the right or left (bottom panel). A difference between the Right-bias group (blue line) and Left-bias (redline) group can once again be seen for both movement types. Error bars show \pm 1 standard error of the mean. (For interpretation of the references to colour in this figure legend, the reader is referred to the web version of this article.)

(start positions: $t[10]=-2.41, p=0.074$; end positions: $t[10]=-2.42, p=0.072)$ and no significant difference from baseline for the right-bias group (start positions: $t$ $[10]=0.60, \quad p=0.56 ;$ end positions: $t[10]=0.95$, $p=0.36)$.

During the Washout-phase, during which participants once again performed 30 postural movements under normal (unbiased) visual feedback conditions, COP position associated with movement start and end position returned to baseline (close to 0 ) for both visual bias groups (Fig. 5). While COP values were very similar among all groups and conditions for the movement start positions (Fig. 5, left panel), some residual effects of the visual manipulation can be seen 
to remain for the movement end positions (Fig. 5, right panel), though the magnitude of the COP differences remained small.

These effects were confirmed using a pair of 3-way ANOVAs. For movement start positions, no main effect of bias GROUP, movement DIRECTION, or TIME (first 10 vs. final 10 trials in the Washout phase) was found (GROUP: $F[1,20]=0.018, p=0.89$; DIRECTION: $F$ $[1,20]=1.42, \quad p=0.25 ; \quad$ TIME: $\quad F[1,20]=0.01$, $p=0.93$ ). Additionally, none of the 2- or 3-way interaction effects were significant (GROUP $\times$ DIRECTION: $\quad F[1,20]=0.21, \quad p=0.89 ; \quad$ GROUP $\times$ TIME: $F[1,20]=0.02, p=0.89$; DIRECTION $\times$ TIME: $F$ $[1,20]=0.12, \quad p=0.73 ; \quad 3$-Way: $\quad F[1,20]=0.027$, $p=0.87$ ). For the movement end positions, no main effect of GROUP $(F[1,20]=0.15, p=0.71)$ or TIME ( $F$ $\{1,20]=0.076, p=0.79)$ was observed, however a main effect of DIRECTION was found $(F[1,20]=26.2$, $p$ < 0.01). Furthermore, a significant 2-way interaction between GROUP and DIRECTION $(F[1,20]=7.01$, $p<0.05)$ and between GROUP and TIME (F[1.20] $=8.73, \quad p<0.01)$ was observed. The interaction between DIRECTION and TIME was not significant $(F$ $[1,20]=0.001, p=0.98)$ nor was the 3-way interaction $(F[1,20]=0.01 . p=0.93)$.

The significant interaction effects for the movement end positions during the Washout phase were examined further using post hoc pairwise comparisons. The results are shown in Table 1. With the stricter criteria associated with such tests, no significant differences were found between the two-bias GROUPs for either of the two movement DIRECTIONS, and at either of the two TIMES. What does emerge, however, is a reliable effect of movement direction (similar to that observed during the Adaptation phase), that remains statistically reliable for the Left-bias group, but not the Right-bias group.

\section{Change in COP during quiet standing following Adaptation and Washout phases}

Immediately following the Adaptation phase, participants exhibited systematic changes in their quiet standing posture relative to baseline. For the left-bias group, the mean mediolateral COP while standing was located $5.18 \mathrm{~mm}(2.60 \mathrm{SE})$ to the left of the baseline (reference) location, while in the right-bias group, the average COP was located $5.43 \mathrm{~mm}$ (3.89 SE) to the right (Fig. 6, left panel). Mean COP following the Washout phase returned closer to the reference position, averaging $0.91 \mathrm{~mm}$ (3.05 SE) for the left-bias group and $0.40 \mathrm{~mm}$ (3.38 SE) for the right-bias group. In contrast with the mediolateral changes, anteroposterior COP showed no reliable difference between the bias groups following both the adaptation and washout phases (Fig. 6, right panel). A 2-way ANOVA examining mediolateral COP at the three phases confirmed the interaction effect $(F$ $[2,40]=10.17, p<0.001)$, with no reliable main effects of bias direction $(F[1,20]=2.84, p=0.11)$ or experimental phase $(F[2,40]=0.161, p=0.85)$. Posthoc comparisons between the left- and right-bias groups show a significant difference only for the Postadaptation phase $(t[20]=2.98, p<0.01)$. Furthermore,
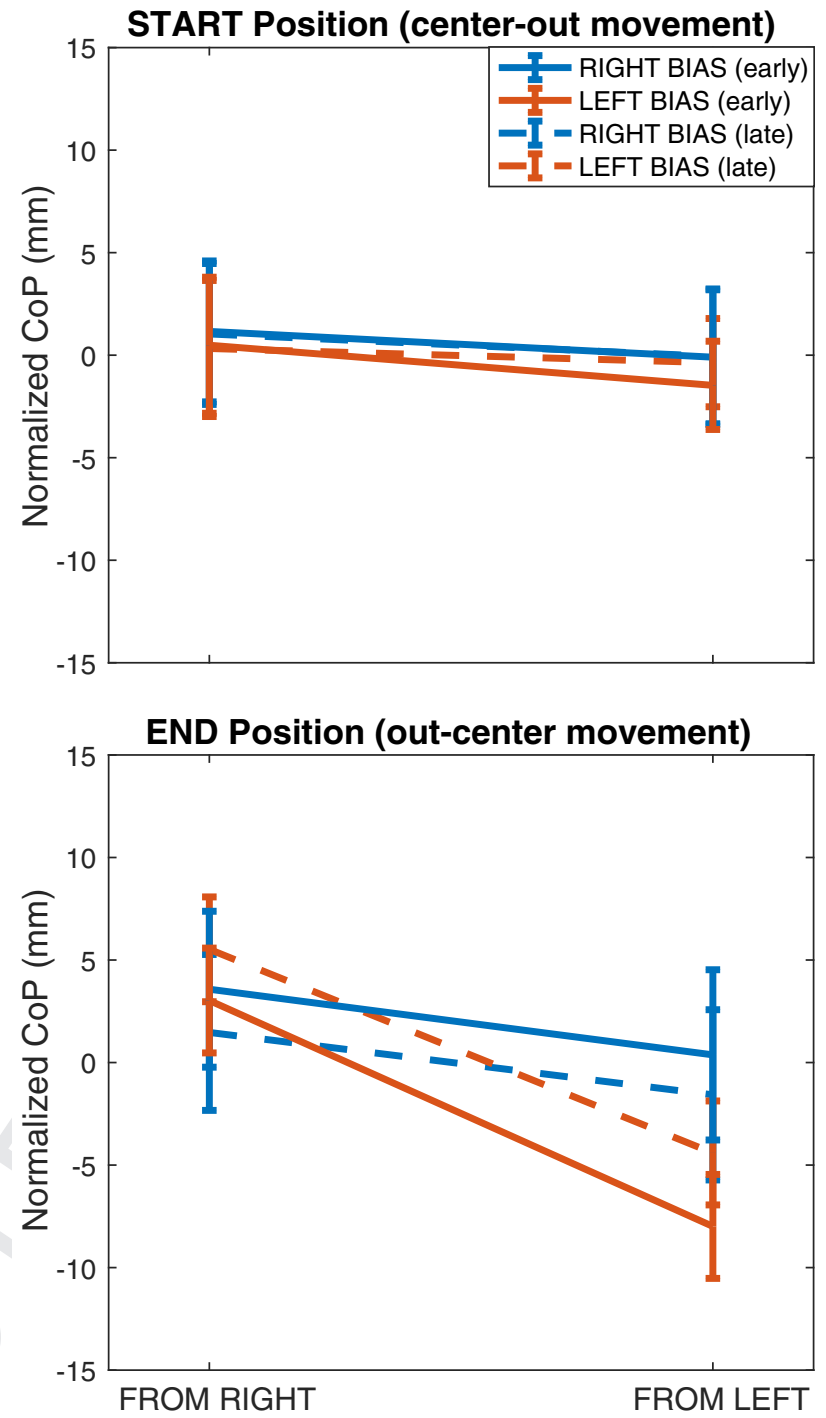

Fig. 5. WASHOUT PHASE (unbiased visual feedback): Mean normalized COP position at the center-base location, observed at the start of center-out movements to the left or right (top panel), and at the end of movements from the right or left (bottom panel). The Dashed lines show COP early during the Washout phase (averaged over the first 10 movements). The Solid lines show COP late in the Washout phase (final 10 movements). Error bars show \pm 1 standard error of the mean.

within this phase, post hoc comparisons examining the COP change (relative to baseline) for each visual-bias direction separately showed a significant effect for the right-bias group $(t[10]=3.10, p<0.05)$ and the leftbias group $(t[10]=-2.96, p<0.05)$.

As expected, an ANOVA examining COP changes along the anteroposterior axis revealed no significant interaction effect $(F[2,40]=1.33, p=0.27)$ and no main effects of bias direction $(F[1,20]=0.064$, $p=0.80)$ or experimental phase $(F[2,40]=0.397$, $p=0.67)$.

\section{DISCUSSION}

The present study addressed the question of whether an asymmetry in whole-body postural control could be 
Table 1. Post-hoc pair-wise comparisons (t-tests with Holm-Bonferroni correction) examining the significant GROUP $\times$ DIRECTION and GROUP $\times$ TIME interaction effects for movement end positions during the Washout phase.

\begin{tabular}{|c|c|c|c|}
\hline \multirow{2}{*}{$\begin{array}{l}\text { TIME } \\
\text { Early }\end{array}$} & \multirow{2}{*}{$\begin{array}{l}\text { DIRECTION } \\
\text { From Right }\end{array}$} & \multicolumn{2}{|c|}{$\begin{array}{l}\text { GROUP EFFECT (Right-bias vs. } \\
\text { Left-bias) }\end{array}$} \\
\hline & & $\mathrm{t}(20)=0.13, p=0.89$ & (NS) \\
\hline & From Left & $\mathrm{t}(20)=1.54, p=0.14$ & (NS) \\
\hline \multirow[t]{2}{*}{ Late } & From Right & $\mathrm{t}(20)=0.88, p=0.39$ & (NS) \\
\hline & From Left & $\mathrm{t}(20)=0.58, p=0.57$ & (NS) \\
\hline TIME & GROUP & \multicolumn{2}{|c|}{$\begin{array}{l}\text { DIRECTION EFFECT (From } \\
\text { right vs. from left) }\end{array}$} \\
\hline \multirow[t]{2}{*}{ Early } & Right-bias & $\mathrm{t}(10)=1.37, p=0.20$ & (NS) \\
\hline & Left-bias & $\mathrm{t}(10)=8.87, p<0.01$ & ${ }^{*} \mathrm{SIG}^{*}$ \\
\hline \multirow[t]{2}{*}{ Late } & Right-bias & $\mathrm{t}(10)=1.09, p=0.29$ & (NS) \\
\hline & Left-bias & $\mathrm{t}(10)=6.25, p<0.01$ & ${ }^{*} \mathrm{SIG}^{*}$ \\
\hline
\end{tabular}

induced by altering the visual feedback of COP position during a dynamic postural motor control task. Participants successfully carried out target-directed COP lateral displacements under conditions of altered visual feedback, with COP movement onsets and offsets closely matching the $30 \mathrm{~mm}$ lateral bias introduced during the Adaptation phase. Following the period of practice carrying out target-directed postural changes under conditions of altered feedback, a shift in COP was found to persist in movement onsets and offsets, consistent with the visual bias direction, even when no visual feedback was present (i.e., a learning aftereffect). Note however that this after-effect was somewhat small, with the two visual bias directions showing a significant difference between each other, but only a marginally significant difference (for the left-bias group) or a non-significant difference (for the right-bias group) from baseline. Additionally, participants' COP location was observed to be shifted reliably to the left or right (depending on the visual bias direction) during postural quiet standing without visual feedback. These results indicate that, after a brief practice period under altered visual feedback conditions, an adaptive change in the control of whole-body posture had been learned by participants, showing a similar influence on both dynamic postural control and quiet standing. (Note that participants, when questioned at the end of the study, reported having had no awareness of this change during the course of the adaptation procedure.)

In the present study, while the learning after-effect was statistically reliable, its magnitude was small compared to the change in visual feedback used to induce adaptation (approximately $5 \mathrm{~mm}$, or $\sim 15 \%$, of the total perturbation of $30 \mathrm{~mm}$ ). It is not uncommon for the magnitude of motor compensation and learning after-effects to be relatively small, especially following only a brief period of practice, as observed in studies of motor adaptation in limb movements to force-field and visual perturbations (see e.g. Shadmehr et al., 2010, for review) and in speech-motor adaptations to altered auditory feedback (e.g., Houde and Jordan, 1998; Purcell and Munhall, 2006). One limiting factor in the present study may have been the size of the visual targets on
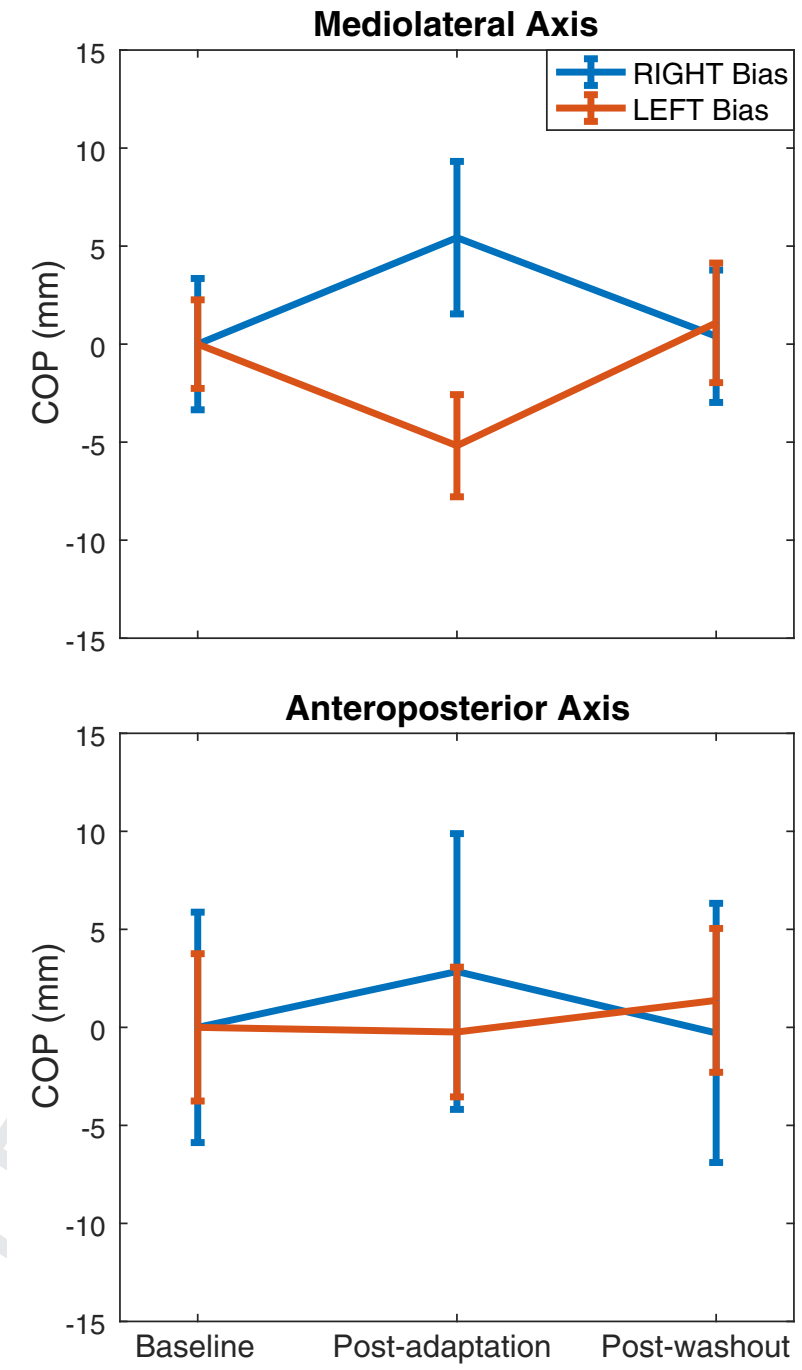

Fig. 6. The mean mediolateral (top panel) and anteroposterior (bottom panel) COP location in the observed during the quiet standing period immediately prior to the Adaptation phase (Standing-Baseline), following the Adaptation phase (Standing-Post-Adaptation), and following the Washout phase (Standing-Post-Washout) for participants in the left-bias (red line) and right-bias (blue line) groups. Error bars show \pm 1 standard error of the mean. (For interpretation of the references to colour in this figure legend, the reader is referred to the web version of this article.)

screen. The use of smaller target regions would have required more accurate shifts in COP, potentially enhancing the magnitude of the sensorimotor adaptation effect. Another limiting factor may have been the use of a simple, 2-D representation of body posture and rectangular targets on a flat screen in front of participants as visual feedback. While our simple display was successful in inducing reliable sensorimotor adaptation effects, studies of sensorimotor adaptation in upper limb movements have shown improved learning outcomes when the task is implemented using a richer, "naturalistic" representation of the hand compared with a simpler, computer-generated representation (Veilleux and Proteau, 2015). It may therefore be possible to increase the magnitude of the postural learning after-effect through the use of a more detailed 
visual feedback system, in which visual-spatial cues to body position and targets are more richly represented.

An effect of movement direction was apparent in the COP movement offsets (see Fig. 3, right panel), reflecting a tendency for participants to end their postural movement closer to the "near-edge" of the center region (i.e., the edge nearer to where the COP movement started from). In other words, when moving their COP from the right target, participants tended to end the movement closer to the right edge of the center region, and when moving from the left target, they tended to end the movement closer to the left edge of the center region. It thus appears as if participants were aiming for the closer edge of the center region, rather than the middle or far edge. While the reason for this is unclear, it may have been related to the fact that the center and target regions were displayed as solid red rectangles with a green outer edge appearing when the participant was to perform the next movement. Hence the outer edge of the movement goal region was visually highlighted, and thus may have appeared to the participant as the most visually salient target. Note that the effect of movement-direction did not interact with the effect of visual bias direction, either at the end of the Adaptation phase or during the No-Feedback phase.

It is interesting, though not altogether surprising, that little or no difference in COP was observed between the early and late parts of the Adaptation and Washout phases (i.e., the effect of TIME included in the 3-way ANOVAs). During the Adaptation phase, the $3-\mathrm{cm}$ bias in visual-feedback toward the right or left was introduced gradually over 60 trials in order to reduce awareness of the change (and hence minimize the use conscious motor strategies). This is consistent with how sensory feedback manipulations have been carried out in numerous studies of limb motor adaptation (e.g., Wolpert et al., 1995; Cressman and Henriques, 2015). Because of the gradual change, it was predicted that participants would effectively "track" the visual shift and achieve nearly complete compensation by the end of the ramping on of the manipulation. This prediction was confirmed by the lack of any observed main or interaction effect of TIME in the analysis of COP in the Adaptation phase.

In the Washout phase, there is some limited evidence of a preserved effect of the visual feedback manipulation, in particular during the first 10 trials (the "early" part). This effect revealed itself as a significant 2-way interaction between GROUP and TIME in the analysis of movement end positions (Fig. 4, right panel). While the interpretation of this interaction is evident from the observed mean differences (showing a larger difference between groups early in the phase compared with the end of the phase), the GROUP effect was subtle and failed to reach significance in post hoc testing at either the early or late TIME. The lack of clear COP adaptation effects in the Washout phase may have been due, in part, to the presence of intervening tasks (the No-Feedback movement condition and the Postadaptation quiet-standing task) between the end of the
Adaptation phase and the beginning of the Washout phase.

The only effect that remained significant under post hoc testing was that of movement DIRECTION: a trend for COP to differ at movement end-points for movements originating from the left vs. from the right. This effect follows the same pattern as that observed for movement end-points during the Adaptation phase (Fig. 3, right panel), however in this case the effect was stronger (and only significant) for the Left-bias group than the Right-bias group. This GROUP $\times$ DIRECTION interaction effect reflects a subtle asymmetry in the way in which the visual bias direction interacted with the direction of movement that was not evident in the Adaptation and No-Feedback phases of the study. Future studies, using different visuomotor manipulations or postural control tasks, may help us better understand the origin of such asymmetries.

In studies of visuomotor adaptation of upper-limb pointing movements to altered visual feedback, sensorimotor learning is believed to reflect, in part, an updating of participants' "internal models" (or mappings) relating motor commands to their sensory consequences - internal representations believed to be central to the planning and control of goaldirected movements (see Kawato, 1999 and Wolpert et al., 2011, for review). In the present study, motor adaptation to changes in visual feedback of COP may similarly involve an updating of predictive internal models, however the precise nature of such changes remains unclear. Models of postural control have highlighted possible roles for both feedback control (e.g., Peterka, 2002) and predictive, feed-forward control (e.g., Morasso et al., 1999). Both control processes are compatible with the current behavioral results. The observed changes in postural control may reflect an updating of internal models relating postural motor commands to their upcoming sensory consequences (i.e., forward models), facilitating the accurate on-line detection and correction of postural deviations (feedback control). The observed motor learning may also reflect changes to internal models relating desired postural outcomes to their underlying motor commands (i.e., inverse models), facilitating accurate feed-forward (predictive) control. Further study, including more varied postural tasks coupled with physiological measures (e.g., EMG) and neuroimaging (e.g., EEG), may ultimately help elucidate the neural mechanisms underlying the behavioral responses observed here.

Two prior studies have shown that it is possible to alter postural control parameters through systematic changes in visual feedback, though the manipulations and resulting postural changes differed in important ways from the present study. In a series of studies involving left-hemiparetic patients, Tilikete et al. (2001) investigated the effect of a visual horizontal prismatic shift on postural imbalance during the performance of upper-limb reaching movements. Following a brief period of practice performing reaching movements 
under visually altered conditions, participants were not only observed to have adapted control of the upper limbs to the change in visual input, but also their wholebody postural control during the reaching task. For example, in adapting to a visual displacement toward the right, participants compensated by not only reaching further to the left with their arm, but also by adjusting their entire body orientation in the same direction. This result was subsequently replicated in a group of healthy participantss (Michel et al., 2003). The authors noted that while the result may have arisen because of sensorimotor recalibration, it more likely reflected a change in participants' higher level cognitive representation of external space relative to the body. This interpretation is supported by the results of numerous prior studies in which healthy participants, following adaptation to prism-shifted visual input, were found to alter performance on a range of neuropsychological tasks including mental imagery (Rode et al., 1999), object recognition (Rossetti et al., 1999) and perceptual line bisection (simulating a form of spatial neglect; Colent et al., 2000).

There are a number of important differences between the sensorimotor adaptation procedure used in the present study and the use of prism glasses in these prior studies, specifically related to the scope of the visual manipulation and the likely mechanism of adaptation. Prism glasses systematically alter perception of the entire visual environment relative to the participant. This explains why, in a task focusing on target-directed arm movements, compensatory adjustments were observed not only in the control of the upper limbs, but in the orientation of the entire body. In contrast, the present study involved a shift in visual feedback related uniquely to the participant's COP, with a movement task that focused specifically on changes in body orientation. Furthermore, the manipulation was carried out without altering the global spatial relation between a participant's body position and the world. The visual representation of the movement start and end location remained unchanged, with the "home" position always at the center of the screen and targets located at a fixed distance to the left or right. Only the participant's real COP relative to the visual dot representing COP on-screen was altered, as if a slight change was introduced in the orientation of the forceplate relative to the ground. Another important difference between the manipulation used in the present study and prism glasses was the timing of the perturbation. The feedback shift in the present study was introduced gradually over 60 trials, rather than suddenly as in the case of prism glasses. As such, participants were not aware of either the perturbation or the resulting adaptation (as reported by participants). The highly specific nature of the perturbation and adaptation effects, coupled with their implicit nature, strongly suggests that adaptation in the current study arose from a recalibration of vestibular-motor processes, rather than a conscious control strategy possibly linked to a change in higher level cognitive representation of external space relative to the body.
Sensorimotor adaptation has shown some promise as an approach to rehabilitating the control of upper limbs. In one example, the adaptation of pointing movements to a force-field applied by a robotic device has been used in children with primary dystonia to improve the control of arm movements (Casallato et al., 2012). Studies of visuomotor adaptation in reaching have also demonstrated that learning after-effects can offset neurological symptoms such as hemineglect in stroke patients. In a number of studies (Rossetti et al., 1998; Pisella et al., 2002), patients exhibiting left-side neglect underwent training to perform reaching movements during a visual perturbation involving a shift to the right (induced by prism glasses). Following the removal of the glasses, a motor learning after-effect led the patients to point toward their neglected left side, with effects that persisted in some cases for days following training (considerably longer than other sensory manipulations, such as neck vibration or optokinetic stimulation).

A possible concern regarding the use of sensorimotor adaptation procedures for clinical treatment is that neurological deficits that negatively affect the control of movement may also limit the capacity for motor adaptation and learning. For example, cerebellar lesions have been found to restrict the degree of improvement following practice in a range of motor adaptation procedures, such as walking and arm movements (Maschke et al., 2004; Morton and Bastian, 2006). However in studies examining other clinical populations, a capacity to significantly improve motor performance over repeated practice trials has been found to remain intact. Disorders of the Basal Ganglia, such as Huntington's or Parkinson's Disease, have shown smaller, but nonetheless significant, motor adaptation effects to altered sensory feedback (Contreras-Vidal and Buch, 2003; Mollaei et al., 2013). Cerebral lesions due to stroke may also reduce the rate of adaptation in reaching movements (Patton et al., 2006; Scheidt and Stoeckmann, 2007), but a capacity for motor learning remains, including in adaptation to walking on a split-belt treadmill (e.g., Reisman et al., 2007).

The demonstration in the present study of visuomotor adaptation in postural control may be valuable not only in its potential for direct rehabilitation, but also because it allows clinicians and researchers to determine whether, in a given patient (or clinical group), the central nervous system is able to achieve normalized patterns of motor behavior, if even for a brief period of time. Such observations have been made in patients with locomotor and upper-limb reaching asymmetry following stroke. One group of patients were trained to walk on a splitbelt treadmill in which the walking speed differed for each leg (Reisman et al., 2007). The resulting motor learning after-effect improved the symmetry of locomotion. While not a lasting effect, the result demonstrated that in these patients the nervous system was indeed capable of near-optimal locomotor control. Similarly, training to produce reaching movements under the influence of an externally applied force-field produced a learning after-effect that, for a brief time, improved the direction of reaching movements (Patton et al., 2006), demonstrat- 
ing that such normalized motor patterns were in fact possible for particular patients.

The present study represents a promising demonstration that visually based sensorimotor adaptation focusing specifically on COP can be used to modify postural control. Clinical applications of such procedures would need to be evaluated in future studies involving populations with postural deficits (such as patients with hemiplegia, who often exhibit asymmetric posture), in order to test and optimize the effectiveness of procedures such as those used in the present study.

\section{UNCITED REFERENCES}

Alcantara et al. (2012), Casellato et al. (2012), JacquinCourtois et al. (2013), Lafond et al. (2004), Raymakers et al. (2005), Redding et al. (2005), Redding and Wallace $(2010,2006)$ and Riach and Starkes (1994).

Acknowledgments-This work was supported by grants from the Natural Sciences and Engineering Research Council (NSERCCanada) and the Fond de Recherche du Québec - Santé (FRQS-Quebec).

\section{REFERENCES}

Alcantara CPA, Prado JM, Duarte M (2012) Analysis of the balance control in surfers during the erect posture. Rev Bras Med Esporte 18:318-321.

Bhushan B, Dwivedi CB, Mishra R, Mandal MK (2000) Performance on a mirror-drawing task by non-right-Handers. J Gen Psychol 127:271-277.

Casellato C, Pedrocchi A, Zorzi G, Rizzi G, Ferrigno G, Nardocci N (2012) Error-enhancing robot therapy to induce motor control improvement in childhood onset primary dystonia. J Neuroeng Rehabil 23:46.

Colent C, Pisella L, Bernieri C, Rode G, Rossetti Y (2000) Cognitive bias induced by visuo-motor adaptation to prisms: a simulation of unilateral neglect in normal individuals. NeuroReport 11:1899-1902.

Contreras-Vidal JL, Buch ER (2003) Effects of Parkinson's disease on visuomotor adaptation. Exp Brain Res 150:25-32.

Cressman EK, Henriques DY (2015) Generalization patterns for reach adaptation and proprioceptive recalibration differ after visuomotor learning. J Neurophysiol 114:354-365.

Di Fabio RP, Badke MB (1990) Extraneous movement associated with hemiplegic postural sway during dynamic goal-directed weight redistribution. Arch Phys Med Rehabil 71:365-371.

Fitzpatrick R, McCloskey DI (1994) Proprioceptive, visual and vestibular thresholds for the perception of sway during standing in humans. J Physiol 478:173-186.

Gordon CR, Fletcher WA, Melvill Jones G, Block EW (1995) Adaptive plasticity in the control of locomotor trajectory. Exp Brain Res 102:540-545.

Hendrickson J, Patterson KK, Inness EL, Mcllroy WE, Mansfield A (2014) Relationship between asymmetry of quiet standing balance control and walking post-stroke. Gait Posture 39:177-181.

Houde JF, Jordan MI (1998) Sensorimotor adaptation in speech production. Science 279:1213-1216.

Jacquin-Courtois S, O'Shea J, Luauté J, Pisella L, Revol P, Mizuno K, Rode G, Rossetti Y (2013) Rehabilitation of spatial neglect by prism adaptation: a peculiar expansion of sensorimotor aftereffects to spatial cognition. Neurosci Biobehav Rev 37:594-609.

Kennedy KM, Raz N (2005) Age, sex and regional brain volumes predict perceptual-motor skill acquisition. Cortex 41:560-569.
Lafond D, Corriveau H, Hebert R, Prince F (2004) Intrasession reliability of center of pressure measures of postural steadiness in healthy elderly people. Arch Phys Med Rehabil 85:896-901.

Mansfield A, Danells CA, Inness E, Mochizuki G, Mcllroy WE (2011) Between-limb synchronization for control of standing balance in individuals with stroke. Clin. Biomech. 26:3312-3317.

Marigold DS, Eng JJ (2006) The relationship of asymmetric weightbearing with postural sway and visual reliance in stroke. Gait Posture 23:249-255.

Martin TA, Norris SA, Greger BE, Thach WT (2002) Dynamic coordination of body parts during prism adaptation. J Neurophysiol 88:1685-1694.

Maschke M, Gomez CM, Ebner TJ, Konczak J (2004) Hereditary cerebellar ataxia progressively impairs force adaptation during goal-directed arm movements. J Neurophysiol 91:230-238.

Mollaei F, Shiller DM, Gracco VL (2013) Sensorimotor adaptation of speech in Parkinson's disease. Move Disord 28:1668-1674.

Morasso PG, Schieppati M (1999) Can muscle stiffness alone stabilize upright standing? J Neurophysiol 82:1622-1626.

Morton SM, Bastian AJ (2006) Cerebellar contributions to locomotor adaptations during split-belt treadmill walking. J Neurosci 26:9107-9116.

Nakajima Y (1988) Effects of up-down visual inversion on motor skills. Percept Mot Skills 67:419-422.

Patton JL, Stoykov ME, Kovic M (2006) Evaluation of robotic training forces that either enhance or reduce error in chronic hemiparetic stroke survivors. Exp Brain Res 168:368-383.

Pham QC, Mello MT, Narciso FV, Mônico Neto M, Teixeira CW, Antonietti LS (2014) Robust evaluation of time since awakening using force platform posturography. Rev Bras Eng Bioméd 30:322-329.

Pisella L, Rode G, Farnè A, Boisson D, Rossetti Y (2002) Dissociated long lasting improvements of straight-ahead pointing and line bisection tasks in two hemineglect patients. Neuropsychologia 40:327-334

Pisella L, Rode G, Farne A, Tilikete C, Rossetti Y (2006) Prism adaptation in the rehabilitation of patients with visuo-spatial cognitive disorders. Curr Opin Neurol:534-542.

Purcell DW, Munhall KG (2006) Adaptive control of vowel formant frequency: evidence from real-time formant manipulation. J Acoust Soc Amer 120:966-977.

Raymakers JA, Samson MM, Verhaar HJ (2005) The assessment of body sway and the choice of the stability parameter(s). Gait Posture 21:48-58.

Redding GM, Wallace B (2006) Prism adaptation and unilateral neglect: review and analysis. Neuropsychologia 44:1-20.

Redding GM, Wallace B (2010) Implications of prism adaptation asymmetry for unilateral visual neglect: theoretical note. Cortex 46:390-396.

Redding G, Rossetti Y, Wallace B (2005) Application of prism adaptation: a tutorial in theory and method. Neurosci Biobehav Rev 29:431-444.

Reisman DS, Block H, Bastian AJ (2005) Inter-limb coordination during locomotion: What can be adapted and stored? J Neurophysiol 94:2403-2415.

Reisman DS, Wityk R, Silver K, Bastian AJ (2007) Locomotor adaptation on a split-belt treadmill can improve walking symmetry poststroke. Brain 130:1861-1872.

Riach CL, Starkes JL (1994) Velocity of centre of pressure excursions as an indicator of postural control system in children. Gait Posture 2:167-172.

Ring H, Mizrahi J (1991) Bilateral postural sway in stroke patients: new parameters for assessing and predicting locomotor outcome. J Neurol Rehab 5:175-179.

Rode G, Rossetti Y, Li L, Boisson D (1999) Improvement of mental imagery after prism exposure in neglect: a case study. Behav Neurol 11:251-258.

Rossetti Y, Rode G, Pisella L, Farné A, Li L, Boisson D, Perenin MT (1998) Prism adaptation to a rightward optical deviation rehabilitates left hemispatial neglect. Nature 395:166-169. 
Rossetti Y, Rode G, Pisella L, Farna A, Li L, Boisson D (1999), Sensori-motor plasticity and cognition: prism adaptation can affect various levels of space representation. In: Grealy M Thomson JA (eds) Studies in perception and action. Erlbaum, New York, pp 265-269.

Scheidt RA, Stoeckmann T (2007) Reach adaptation and final position control amid environmental uncertainty after stroke. J Neurophysiol 97:2824-2836.

Shadmehr R, Mussa-Ivaldi FA (1994) Adaptive representation of dynamics during learning of a motor task. J Neurosci 14:3208-3224.

Shadmehr R, Smith MA, Krakauer JW (2010) Error correction, sensory prediction, and adaptation in motor control. Annu Rev Neurosci 33:89-108.
Shumway-Cook A, Woollacott MH (2007) Motor control: translating research into clinical practice. 4th ed. Lippincott Williams \& Wilkins.

Veilleux LN, Proteau L (2015) Prism adaptation in virtual and natural contexts: evidence for a flexible adaptive process. Q J Exp Psychol 68:1168-1182.

Weber KD, Fletcher WA, Gorden CR, Melvill Jones G, Block EW (1998) Motor learning in the "podokinetic" system and its role in spatial orientation during locomotion. Exp Brain Res 120:377-385.

Winter DA (1995) Human balance and posture control during standing and walking. Gait Posture 3:93-214.

Wolpert DM, Ghahramani Z, Jordan MI (1995) Are arm trajectories planned in kinematic or dynamic coordinates? An adaptation study. Exp Brain Res 103:460-470.
996

(Received 15 March 2017, Accepted 17 May 2017)

(Available online $x x x x$ ) 